\title{
Pain and autonomic dysfunction in patients with sarcoidosis and small fibre neuropathy
}

\author{
M. Bakkers • C. G. Faber $\cdot$ M. Drent . \\ M. C. E. Hermans $\cdot$ S. I. van Nes $\cdot$ G. Lauria • \\ M. De Baets · I. S. J. Merkies
}

Received: 2 May 2010/Revised: 22 June 2010/ Accepted: 5 July 2010/Published online: 20 July 2010

(C) The Author(s) 2010. This article is published with open access at Springerlink.com

\begin{abstract}
Small fibre neuropathy (SFN) has been demonstrated in sarcoidosis. However, a systematic analysis of neuropathic pain and autonomic symptoms, key features of SFN, has not been performed. Clinimetric evaluation of pain and autonomic symptoms using the neuropathic pain scale (NPS) and the modified Composite Autonomic Symptoms Scale (mCOMPASS) was used in sarcoidosis patients for this study. A total of 91 sarcoidosis patients ( $n=23$ without SFN symptoms, $n=43$ with SFN
\end{abstract}

M. Bakkers $(\square)$ C. G. Faber · M. C. E. Hermans ·

I. S. J. Merkies

Department of Neurology, Maastricht University Medical Centre, P.O. Box 5800, 6202 AZ Maastricht, The Netherlands

e-mail: m.bakkers@mumc.nl

\section{Drent}

Department of Respiratory Medicine, Interstitial Lung Disease Care Team, Maastricht University Medical Centre,

P.O. Box 5800, 6202 AZ Maastricht, The Netherlands

S. I. van Nes - I. S. J. Merkies

Department of Neurology, Erasmus Medical Centre Rotterdam, P.O. Box 2040, 3000 CA Rotterdam, The Netherlands

\section{G. Lauria}

Neuromuscular Diseases Unit, IRCCS Foundation,

National Neurological Institute "Carlo Besta",

via Celoria 11, 20133 Milan, Italy

M. De Baets

Department of Neuroscience, School for Mental Health and Neuroscience, Universiteitssingel 50,

6229 ER Maastricht, The Netherlands

I. S. J. Merkies

Department of Neurology, Spaarne Hospital, Spaarnepoort 1,

2134 TM Hoofddorp, The Netherlands symptoms but normal intraepidermal nerve fibre density (IENFD), $n=25$ with SFN symptoms and reduced IENFD) were examined. NPS and mCOMPASS were assessed twice (reliability studies). Severity of pain was compared between the subgroups. Correlation between NPS and a visual analogue pain scale (VAS) was assessed (validity studies). Healthy controls $(n=105)$ completed the mCOMPASS for comparison with patients' scores. Patients with sarcoidosis, SFN complaints, and reduced IENFD demonstrated more severe pain scores on the NPS. The mCOMPASS differentiated between subjects with and without SFN symptoms. A significant correlation was obtained between the NPS and VAS, indicating good construct validity. Good reliability values were obtained for all scales. The use of the NPS to evaluate SFN symptoms is suggested, as it shows differences between patients with SFN symptoms with normal or reduced IENFD values. The mCOMPASS might be used to select patients for further testing.

Keywords Pain - Neuropathic pain scale . Autonomic dysfunction - Composite Autonomic Symptoms Scale $\cdot$ Sarcoidosis $\cdot$ Small fibre neuropathy

\section{Introduction}

Small fibre neuropathy (SFN) is a disorder with selective involvement of small-calibre myelinated (A $\delta$ ) and unmyelinated $(\mathrm{C})$ nerve fibres, characterised by neuropathic pain and autonomic symptoms. Diagnosis is usually made on the basis of clinical features (neuropathic pain, loss of pinprick and temperature sensation), in combination with abnormal specialized tests such as the assessment of intraepidermal nerve fibre density (IENFD) in skin biopsy, 
temperature sensation tests for sensory fibres, and sudomotor and cardiovagal testing for autonomic fibres [1].

Pain is a frequent complaint in sarcoidosis, an inflammatory multisystem disorder of unknown aetiology which may involve any part of the nervous system [2], and pain is related to lower quality of life [3]. We recently demonstrated SFN to be frequent in sarcoidosis [4]. However, neither the type or intensity of pain, nor the extent to which autonomic symptoms may occur in SFN in sarcoidosis have been studied systematically in this condition. The current study describes the various aspects of pain and autonomic symptoms in patients with sarcoidosis using the neuropathic pain scale (NPS) and a modified Composite Autonomic Symptoms Scale (mCOMPASS), and assesses validity and reliability of these outcome measures.

\section{Participants and methods}

\section{Participants}

One hundred and five healthy volunteers were recruited from hospital personnel, relatives and friends of patients, at sports accommodations, and informal meetings for the elderly. Inclusion criteria were: no pain or other neurological complaints, no history of alcohol abuse, no diseases that may cause sensory deficit or pain sensation, and normal findings at neurological examination [6].

Patients referred to the Maastricht Sarcoidosis Management Centre, a referral centre for sarcoidosis in The Netherlands, were screened for eligibility. We included 91 patients in the study. Inclusion criteria were: diagnosis of sarcoidosis [5], lucid consciousness, no alcohol abuse, no usage of immunosuppressant drugs in the past 6 months, no diseases that may cause sensory deficit, and no signs of central nervous system involvement or large fibre neuropathy (no abnormal nerve conduction studies). After inclusion, patients were categorised as either "no SFN", or, when having SFN symptoms as "possible SFN". A patient was classified as having SFN symptoms when he or she reported at least one of the following symptoms, not otherwise explained: burning pain in extremities, dry mouth or eyes, changes in sweating, flushes, gastrointestinal dysfunction (constipation, diarrhea), cardiac complaints (palpitation, dizziness at standing up), urogenital dysfunction (sexual dysfunction, incontinence) [6].

Scale selection

The NPS was designed to assess distinct pain qualities associated with neuropathic pain and has been used in peripheral neurological conditions [7]. The questionnaire rates ten different aspects of pain on a numerical $0-10$ scale. Addressed are intensity and unpleasantness of pain in general; intensity of sharpness, hotness, dullness, coldness, skin sensitivity, itching; and intensity of surface and deep pain.

The horizontal visual analogue pain scale (VAS) is a 10$\mathrm{cm}$ horizontal line, depicting no pain at the left and worst pain ever at the right side. Patients mark their pain intensity at the line. It is considered to reliably assess a patient's experience [8].

The original Composite Autonomic Symptom Scale (COMPASS), validated in patients with autonomic failure and non-autonomic neuropathies, correlates well with autonomic function tests [9]. Its 73 questions concern different aspects of the autonomic system: orthostatic intolerance and reflex syncope, secretomotor, vasomotor, pupillomotor, urogenital, gastrointestinal, and sleep difficulties.

Translation and modification

The translation of the NPS and mCOMPASS was performed according to the international guidelines [10]. Some domains of the COMPASS were simplified by a clinimetrician (ISJM), and questions on female sexuality were added, reflecting an equivalent score for male sexuality, resulting in a 65 item modified scale (mCOMPASS), with a sum score of 200 for both men and women.

\section{Skin biopsies}

All participants underwent skin biopsy for intraepidermal nerve fibre density (IENFD) determination according to European guidelines [11]. Biopsies were taken $10 \mathrm{~cm}$ above the lateral malleolus. Normative values were used to determine normal versus impaired IENFD findings [6]. Patients were further divided into subgroups according to the presence of symptoms combined with skin biopsy results: group A, patients without SFN symptoms; B, patients with SFN symptoms but normal IENFD; and C, patients with SFN symptoms and reduced IENFD. We expected those with both symptoms and reduced IENFD to be most affected, IENFD being considered an objective tool to diagnose SFN [11, 12].

Study design and statistics

The study was approved by the medical ethical committee of the Maastricht University Medical Centre (Central Committee for Human Related Research, identifier number p06.0066L/MEC 05-224), in accordance with the ethical standards laid down in the 1964 Declaration of Helsinki. Informed consent was obtained from all participants prior to the study. All patients were examined at the Maastricht 
University Medical Centre. Examination took take place in a comfortable, temperature-controlled room. Questionnaires were provided with written instructions. Healthy controls were requested to complete the mCOMPASS. Data collection, entry, and management were performed using the Teleform automated processing system.

Reliability and validity studies

Patients completed the NPS and mCOMPASS twice, within 2-4 weeks, without having access to their previous answers (test-retest reliability; weighted kappa-statistic $(\kappa)$ measures) [13]. We examined the discriminatory validity of the NPS in the various patients' subgroups in relation to severe pain, defined as a numerical rating score $>5$ on a NPS question (Chi-square test). Correlation studies between the NPS (item pain intensity) and VAS pain scales were also performed (convergent validity of NPS; Spearman's Rank tests). For the mCOMPASS domains, subgroups comparison (one-way ANOVA + Bonferonni corrections) was performed. All analyses were performed using Stata 10.0 for Windows XP.

\section{Results}

From October 2006 to July 2008, 105 healthy controls (54 women and 51 men; mean age 45.1 (SD: 14.6; range:
20-79) years), and 91 sarcoidosis patients (41 women, 50 men; age 45.5 (SD: 9.1; range: $27-70$ ) years) were included in the study. The patients with sarcoidosis were subdivided into three groups: Group A, patients without SFN symptoms $(n=23)$; B, patients with SFN symptoms but normal IENFD $(n=43)$; and C, patients with SFN symptoms and impaired IENFD $(n=25)$.

In group A, $35 \%$ of patients reported severe pain, in group B $65 \%$, in group C $76 \%$. Not only intensity of pain, but also various other qualities of pain were significantly more severe in group $\mathrm{C}$ compared to the other subgroups: "sharpness", "burning", “dullness", "unpleasant pain sensation", and "intense surface pain" (Fig. 1). More severe pain was also seen for the qualities "sensitivity of skin" and "intense deep pain" sensations, but the differences did not reach significance.

Good correlation was obtained between the NPS and VAS pain scales (NPS (intensity) versus VAS: Spearman's rho 0.73).

The mCOMPASS demonstrated higher scores (more autonomic dysfunction related symptoms) for all domains and sum scores in the patients with SFN complaints (groups $\mathrm{B}$ and $\mathrm{C}$ ) compared to group A and healthy controls, but did not discriminate between the groups B and C (Fig. 2). For the sexual disorders and syncope domains, no discrimination between the subgroups and healthy controls was obtained.

Reliability studies showed good test-retest scores for all domains of the NPS and mCOMPASS (weighted kappa
Fig. 1 Graphical representation of several items of the neuropathic pain scale (NPS) in patients with sarcoidosis, those pain qualities with significant differences between subgroups are shown $(p \leq 0.04)$. Bars show percentage of patients in the three patient groups with (in blue) no, (red) minor to moderate or (green) severe pain. Group A, sarcoidosis patients without small fibre neuropathy (SFN) symptoms. Group B, sarcoidosis patients with SFN symptoms, but normal intraepidermal nerve fibre density (IENFD). Group C, sarcoidosis patients with SFN symptoms and impaired IENFD

\section{Neuropathic Pain Scale}

No pain sensation $($ score $=0$ )

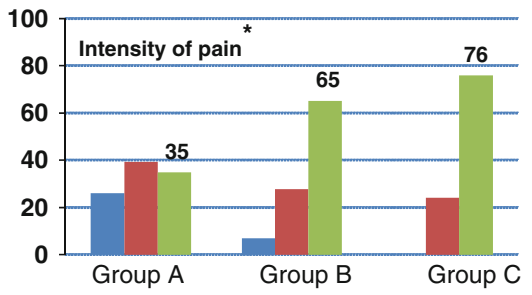

${ }^{*} p \leq 0.04$
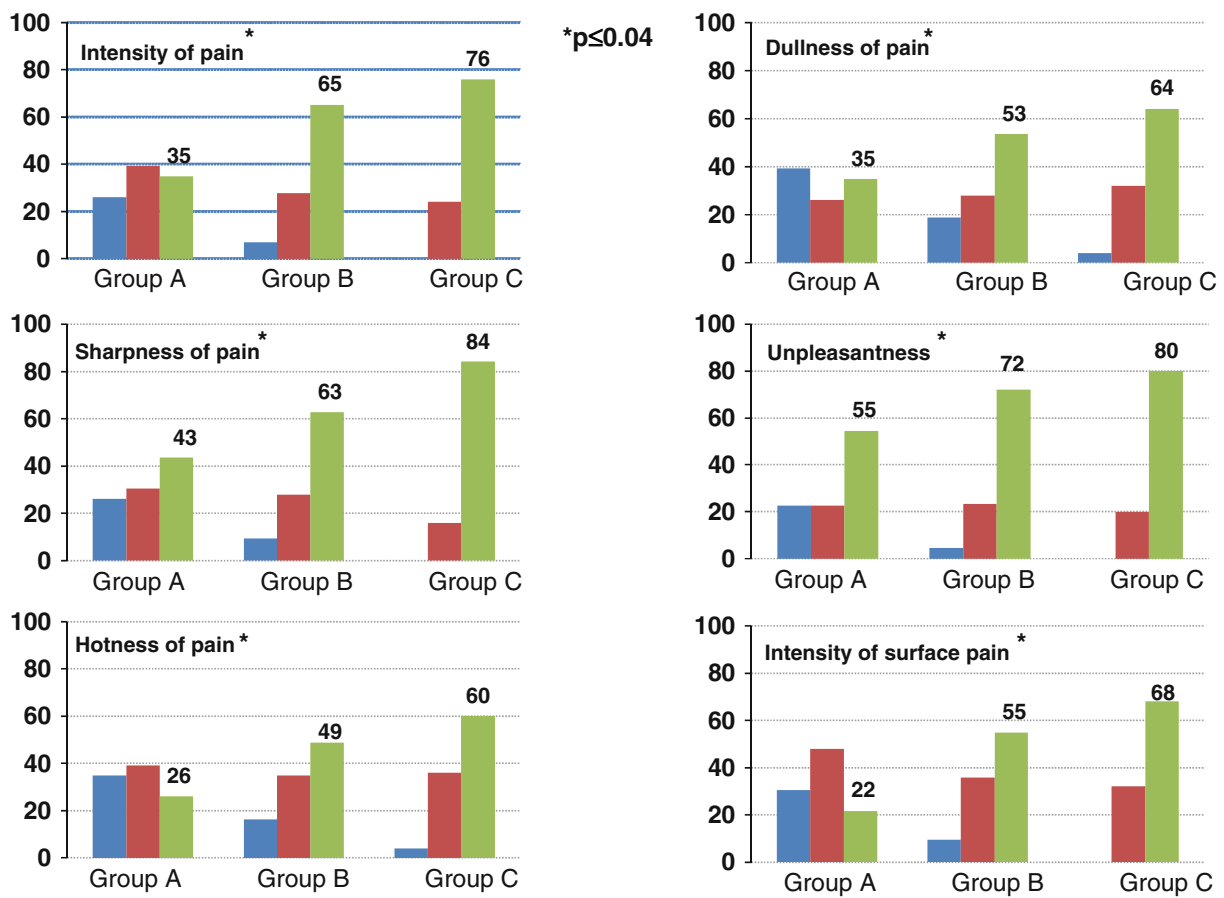


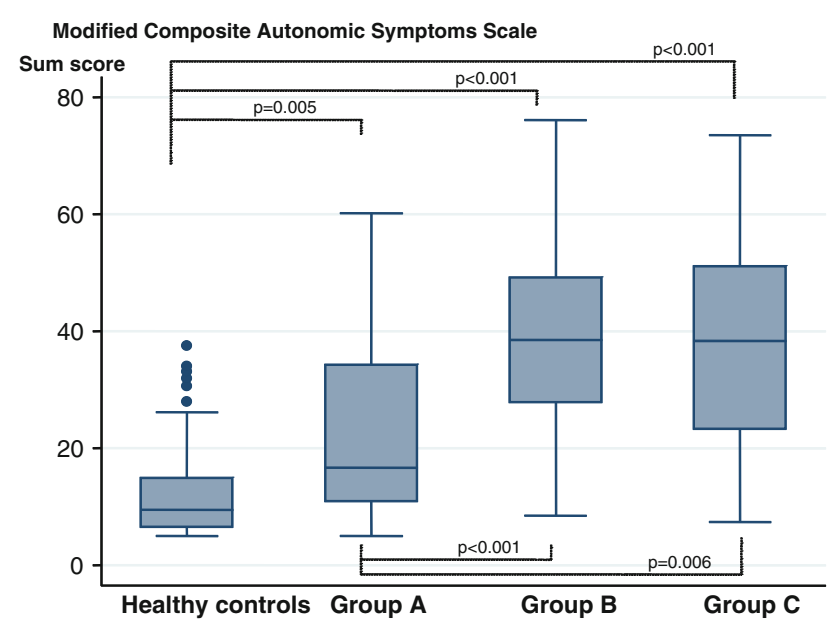

Fig. 2 Box plots showing median and interquartile ranges of the modified version of the Composite Autonomic Symptoms Scale (mCOMPASS) sum scores for healthy controls and various subgroups of patients with sarcoidosis. Comparison of subgroups by one way Anova + Bonferonni corrections. Group A $(n=23)$, patients without small fibre neuropathy (SFN) symptoms. Group B $(n=43)$, patients with SFN symptoms and normal intraepidermal nerve fibre density (IENFD). Group C $(n=25)$, patients with SFN symptoms and reduced IENFD. Between groups $\mathrm{B}$ and $\mathrm{C}$, differences were not significant

0.60-0.95), except for the mCOMPASS syncope domain (0.39). The mCOMPASS sum score had a kappa of 0.88 .

\section{Discussion}

The current study describes the various aspects of pain and autonomic dysfunction in patients with sarcoidosis using the NPS and the mCOMPASS. Various neuropathic pain modalities measured with the NPS were more severe in patients with SFN complaints and even more so in those with reduced IENFD values (group C). This tool may be of use in interventional studies; for example, in evaluating the efficacy of disease-modifying therapies [14].

As expected, higher mCOMPASS scores were found in patients with SFN symptoms. Nevertheless, no significant difference in sum scores was seen between group B and C. Autonomic nerve fibres are of a different type compared to the measured ones in skin, and agreement between IENFD and autonomic deficits varies [15]. Autonomic tests can be very time consuming though, with a high burden to patients [16]. Therefore, the use of this scale is suggested to select patients in whom further testing for SFN might be warranted.

The NPS correlated well with the VAS, indicating good construct validity. Both the NPS and the mCOMPASS demonstrated good test-retest reliability.

There are some methodological issues that should be addressed. Some caution is requested when interpreting the results, since the sample sizes of the various subgroups were relatively small. Also, we did not exclude patients with painful sarcoidosis related problems such as arthritis. It may have been difficult for patients to differentiate between their pain origins, and it might explain why such a large proportion of patients in group A reports severe pain. However, the qualities regarded as typically neuropathic (sharp, hot, superficial pain) were especially more severe in patients with SFN symptoms and reduced IENFD. Furthermore, the mCOMPASS is a composite ordinal scale based on the classic test theory, which may have disadvantages [17].

In conclusion, the neuropathic pain scale shows differences between patients with SFN symptoms with normal or reduced IENFD values, and its use is therefore suggested in future (interventional) studies of this condition. The presence of autonomic symptoms as measured by the mCOMPASS might be used to select patients for further testing with regard to possible SFN.

Conflict of interest statement The first author and the described study were funded by the "Profileringsfonds" [062053] of the Maastricht University Medical Centre.

Open Access This article is distributed under the terms of the Creative Commons Attribution Noncommercial License which permits any noncommercial use, distribution, and reproduction in any medium, provided the original author(s) and source are credited.

\section{References}

1. Hoitsma E, Reulen JP, de Baets M, Drent M, Spaans F, Faber CG (2004) Small fiber neuropathy: a common and important clinical disorder. J Neurol Sci 227:119-130. doi:10.1016/j.jns.2004.08.012

2. Iannuzi MC, Rybicki BA, Teirstein AS (2007) Sarcoidosis. N Engl J Med 357:2153-2165. doi:10.1056/NEJMra071714

3. Hoitsma E, De Vries J, van Santen-Hoeufft M, Faber CG, Drent M (2003) Impact of pain in a Dutch sarcoidosis patient population. Sarcoidosis Vasc Diffuse lung Dis 20:33-39

4. Hoitsma E, Marziniak M, Faber CG, Reulen JP, Sommer C, De Baets M, Drent M (2002) Small fiber neuropathy in sarcoidosis. Lancet 359:2085-2086

5. Hunninghake GW, Costabel U, Ando M, Baughman R, Cordier JF, du Bois R, Eklund A, Kitaichi M, Lynch J, Rizzato G, Rose C, selroos O, Semenzato G, Sharma OP (1999) ATS/ERS/WASOG statement on sarcoidosis American Thoracic society/European Respiratory Society/world Association of Sarcoidosis and other Granulomatous Disorders. Sarcoidosis Vasc Diffuse Lung Dis 16:149-173

6. Bakkers M, Merkies IS, Lauria G, Devigili G, Penza P, Lombardi R, Hermans MC, van Nes SI, De Baets M, Faber CG (2009) Intraepidermal nerve fiber density and is application in sarcoidosis. Neurology 73:1142-1148. doi:10.1212/WNL.0b013e3181bacf05

7. Galer BS, Jensen MP (1997) Development and preliminary validation of a pain measure specific to neuropathic pin: the neuropathic pain scale. Neurology 48:332-338

8. Ohnhaus EE, Adler R (1975) Methodological problems in the measurement of pain: a comparison between the verbal rating scale and the visual analogue scale. Pain 1:379-384 
9. Suarez GA, Opfer-Gehrking TL, Offord KP, Atkinson EJ, O'Brien PC, Low PA (1999) The autonomic symptom profile: a new instrument to assess autonomic symptoms. Neurology 52:523-528

10. Streiner DL, Normal GR (1998) Health measurement scales. A practical guide to their development and use, 2nd edn. Oxford University Press, New York

11. Lauria G, Cornblath DR, Johanssen O, McArthur JC, Mellgren SI, Nolano M, Rosenberg N, Sommer C, European Federation of Neurological Societies (2005) EFNS guidelines on the use of skin biopsy in the diagnosis of peripheral neuropathy. Eur J Neurol 12:747-758

12. Lauria G, Lombardi R (2007) Skin biopsy, a new tool for diagnosing peripheral neuropathy. BMJ 334:116-1159

13. Cohen J (1968) Weighted kappa: nominal scale agreement with provision for scale disagreement or part credit. Psychol Bull 70:213-230
14. Baughman RP, Drent M, Kavuru M et al (2006) Infliximab therapy in patients with chronic sarcoidosis and pulmonary involvement. Am J Respir Crit Care Med 174:795-802. doi:10.1164/rccm.200603-4020C

15. Singer W, Spies M, McArthur J, Low J, Griffin JW, Nickander KK, Gordon V, Low PA (2004) Prospective evaluation of somatic and autonomic small fibers in selected autonomic neuropathies. Neurology 62:612-618

16. Ravits JM (1997) AAEM minimonograph \#48: autonomic nervous system testing. Muscle Nerve 20:919-937

17. DeVellis RF (2006) Classical test theory. Med Care 44(3):S50 S59. doi:10.1097/01.mlr.0000245426.10853.30 